Supporting Information

In-situ nano transformable hydrogel for chemo-photothermal therapy of localized tumors and targeted therapy of highly metastatic tumors

Syed Baseeruddin Alvi ${ }^{a, \neq}$, Rajalakshmi P $S^{a, \neq}$, Nazia Begum ${ }^{b}$, Anil Bankati Jogdand ${ }^{a}$, Bantal Veeresh ${ }^{b}$, Aravind Kumar Rengan ${ }^{a, *}$.

${ }^{a}$ Department of Biomedical Engineering, Indian Institute of Technology Hyderabad, Telangana-502285, India.

${ }^{b}$ Department of Pharmacology, G. Pulla Reddy College of Pharmacy, Hyderabad, Telangana500028, India.

${ }^{\ddagger}$ Authors contributed equally

* Corresponding author, Email: aravind@bme.iith.ac.in 


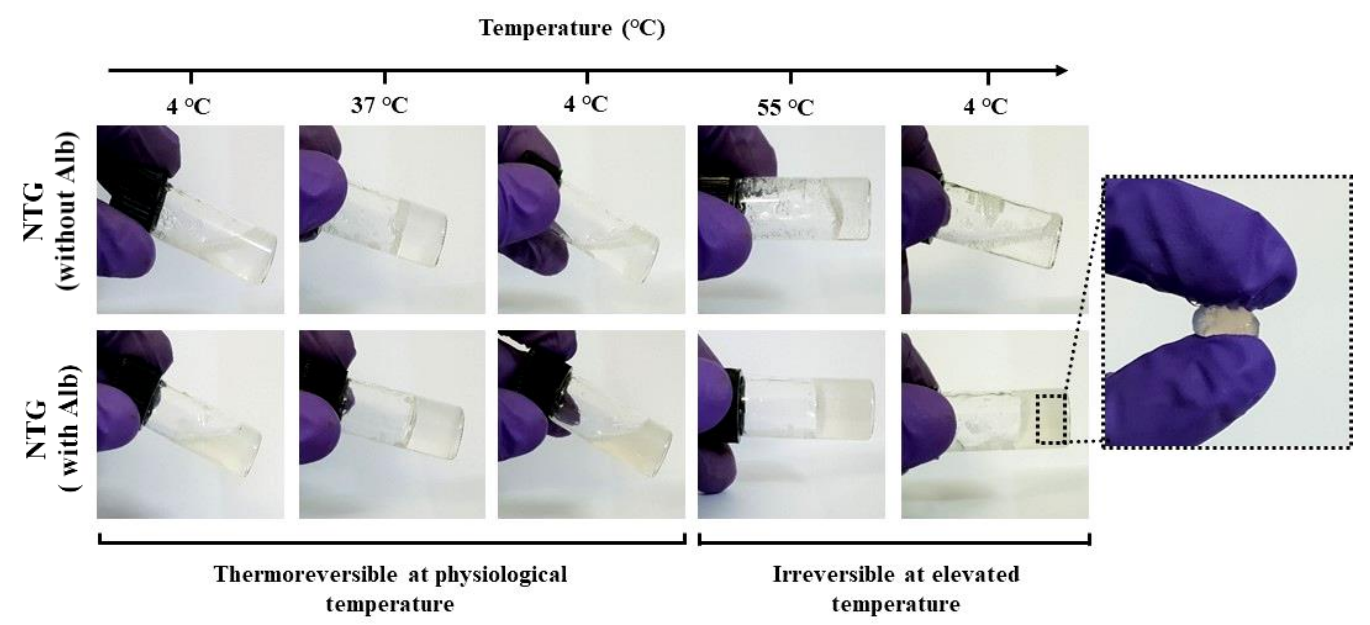

Figure S1: Temperature induced Sol-Gel and Gel-Sol transition of NTG (with/without Alb) at varring temperature 4 ${ }^{\circ} \mathrm{C}, 37^{\circ} \mathrm{C} \& 60^{\circ} \mathrm{C}$.
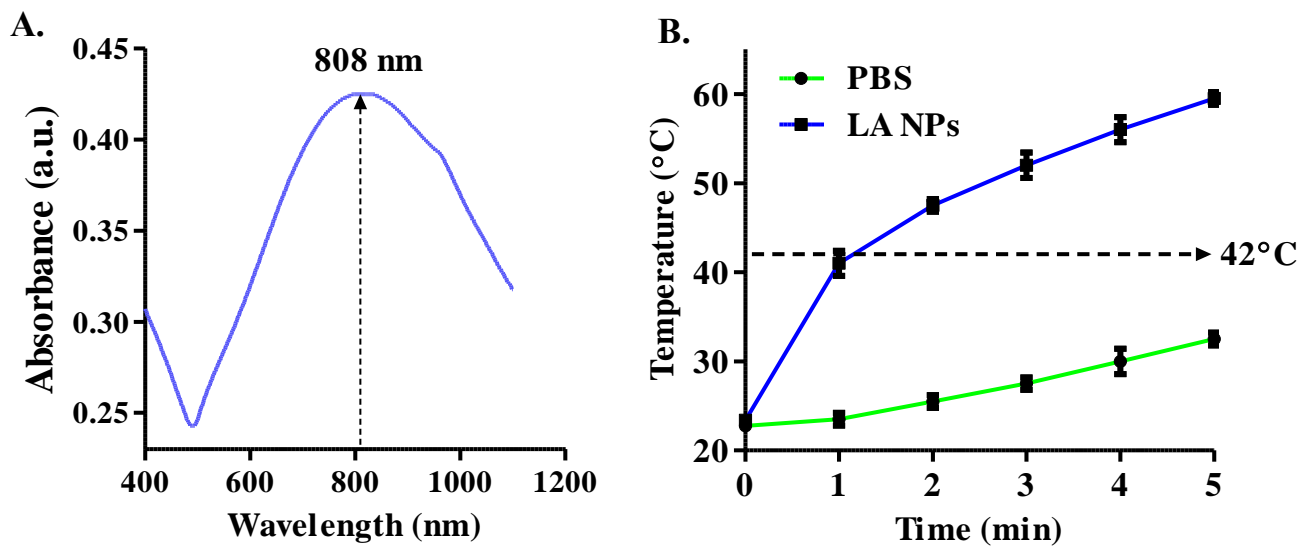

Figure S2: A. UV-Vis spectroscopic analysis of LA-NPs. B. Photothermal transduction of LA NPs when irradiated with NIR light (808 $\mathrm{nm})$. Data is representated as mean \pm SD.

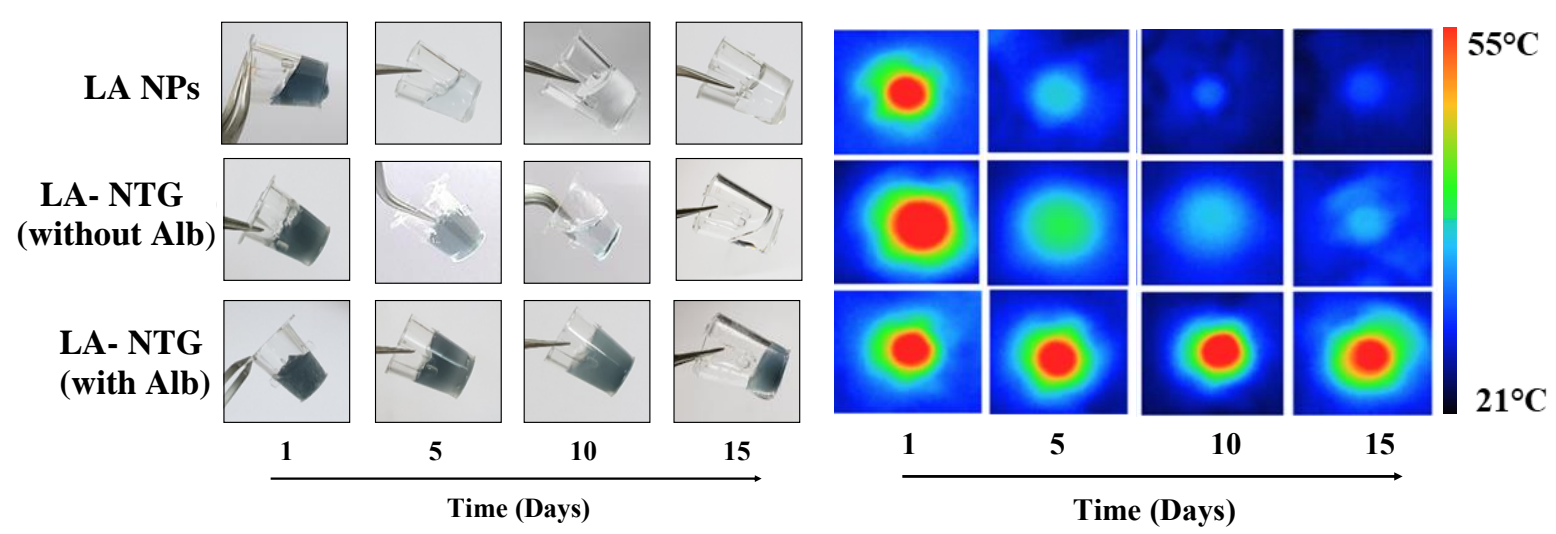

Figure S3: Dissolution of LA-NTG (with/without Alb) and its sustained photothermal transduction efficacy. 


\section{LA-NTG (+L)}
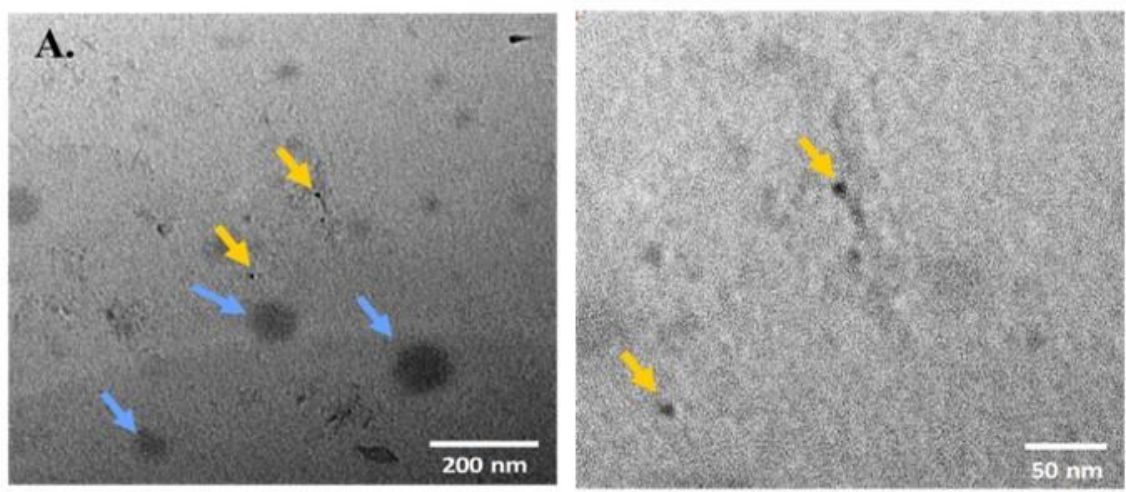

P NPs

PA NPs
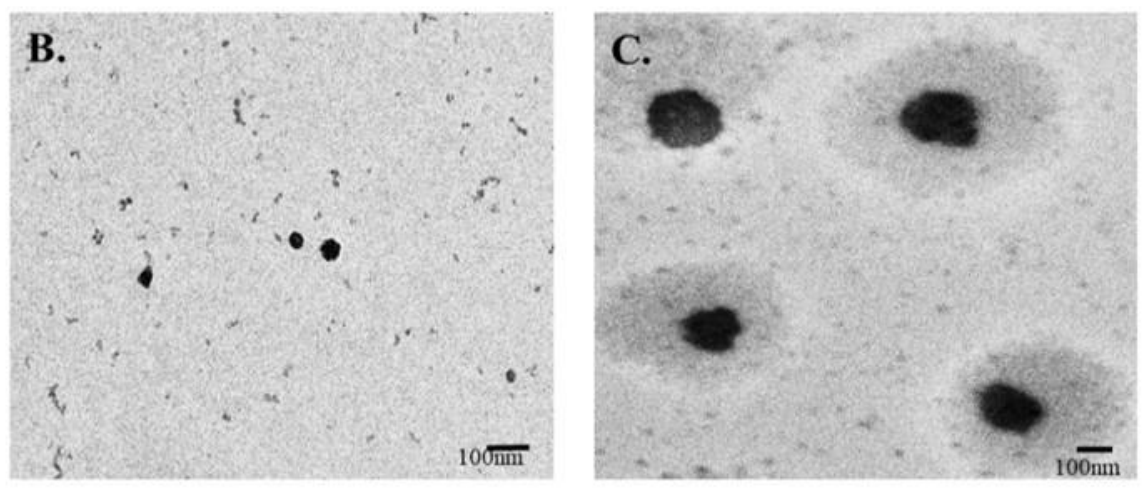

D.

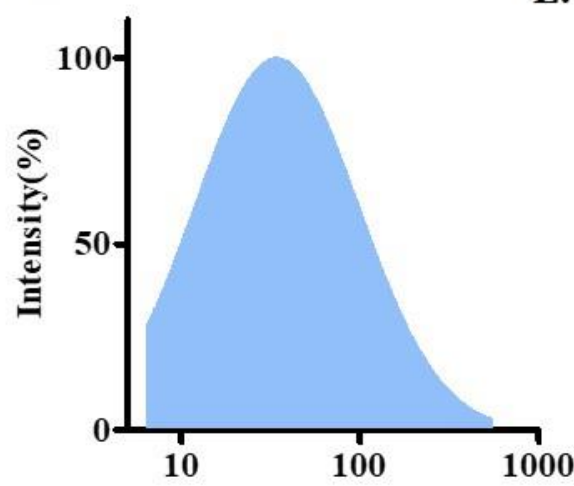

Hydrodynamic diameter (nm)

E.

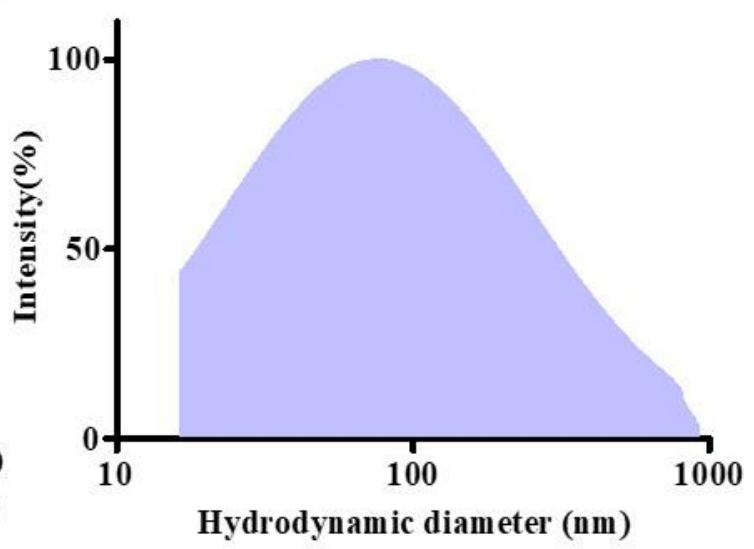

Figure S4: A. TEM imaging of degraded LA NPs (Yellow arrows) following NIR laser irradiation and in situ formed PA NPs (Blue arrows) released from LA-NTG (image from the same sample showing both degraded LA NPs and PA NPs in the same field). B. \& C. P NPs and PA NPs released from NTG without and with Alb rescpectively. D. DLS analysis of P NPs released from NTG (without Alb) E. DLS data of PA NPs without NIR light irradiation. 
A.

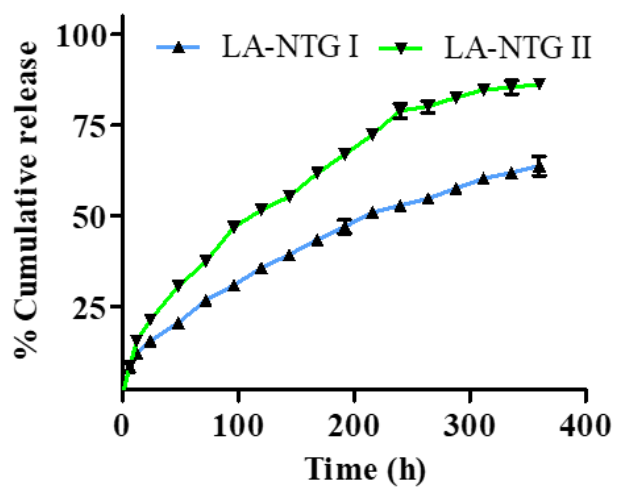

B.

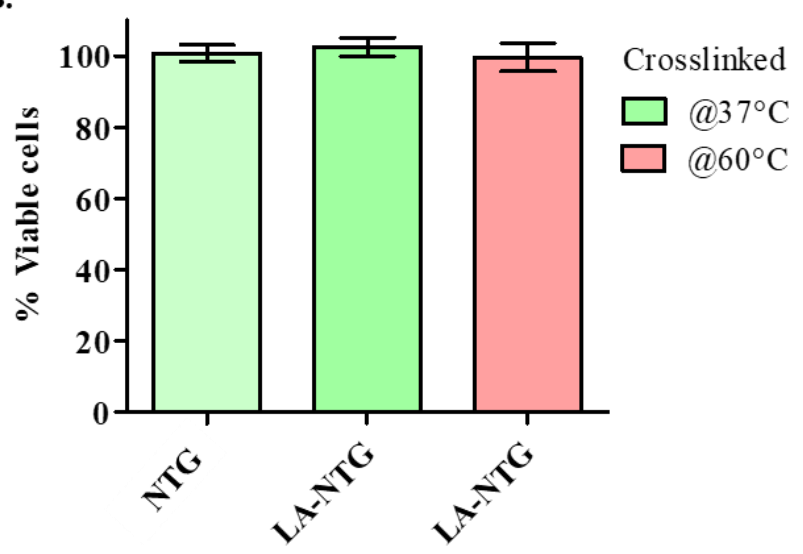

Figure S5: A. NIR light trigerred cumulative release of Dox from LA-NTG I and LA NTG II. B. Biocompatibility of LA-NTG cross-linked at $37{ }^{\circ} \mathrm{C}$ and $60{ }^{\circ} \mathrm{C}$ assessed on NIH 3 T3 cell line. Data is representated as mean \pm SD. 

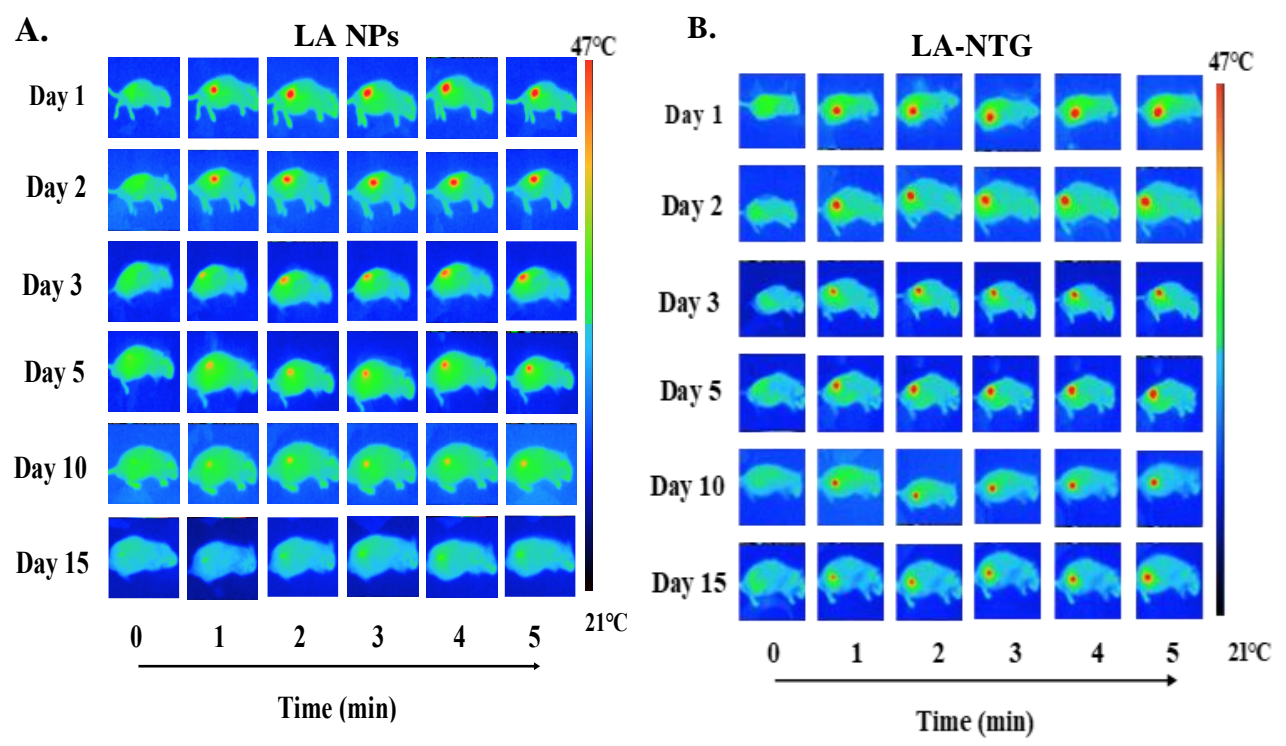

c.
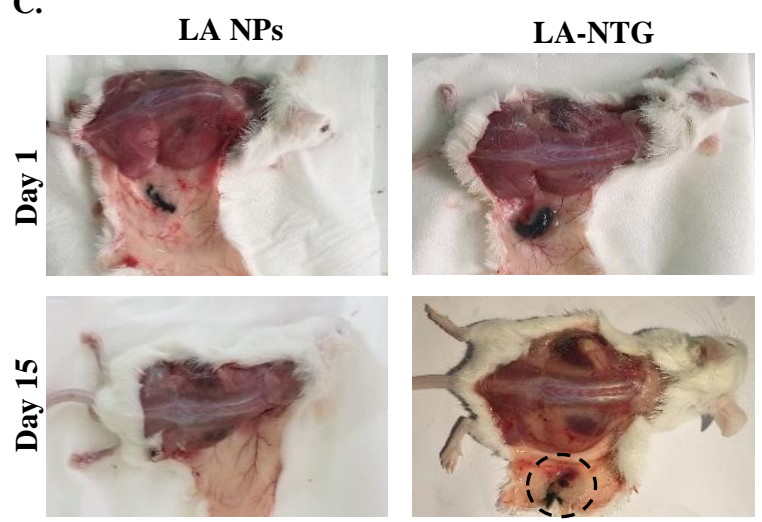

Figure S 6: In-vivo photothermal transduction and sustained photothermal therapy by, A. LA NPs, B. LA-NTG C. Localized retention of LA NPs entrapped in LA-NTG for 15 days. 


\section{Supporting Information}

\section{NIR laser mediated cell cytotoxicity of smart hydrogel}

The NIR laser mediated cell cytotoxicity of the NTG, D-NTG, LA-NTG, DLA-NTG hydrogels were evaluated in melanoma cells (B16F10) using MTT assay and also by live and dead assay. Briefly, $1 \times 10^{5}$ cells were seeded in the lower compartment of the transwell plate and incubated for $24 \mathrm{~h}$. $300 \mu \mathrm{L}$ of each hydrogel was added to the inset (pore size $=3 \mu \mathrm{m}$ ) of the trans-well plate and incubated at $37{ }^{\circ} \mathrm{C}$ for $5 \mathrm{~min}$. The inset containing the cross-linked hydrogel was placed in the trans-well plate and irradiated with laser for $7 \mathrm{~min}$. To evaluate the laser-mediated cell cytotoxicity, MTT assay was performed, and the live and dead assay was performed using FDA (Ex: $450 \mathrm{~nm}$ Em: $515 \mathrm{~nm}$ ) \& PI (Ex: $490 \mathrm{~nm}$ Em: $630 \mathrm{~nm}$ ) to evaluate qualitatively. The experiments were conducted in triplicates.

The DLA-NTG was then evaluated for photothermal chemotherapy on murine melanoma cell line (B16F10). The MTT assay confirmed the enhanced cytotoxicity of the DLA-NTG treated group to about $83.57 \pm 2.5 \%$, whereas, in the LA-NTG treated group, the cytotoxicity was about $54.47 \pm 0.36 \%$ following NIR laser irradiation (Figure S 7). The same was confirmed by live/dead assay (Figure S 8). The localized and enhanced cytotoxicity observed in the DLANTG treated group was due to the combination of both photothermal therapy and chemotherapy. 
B.

A.
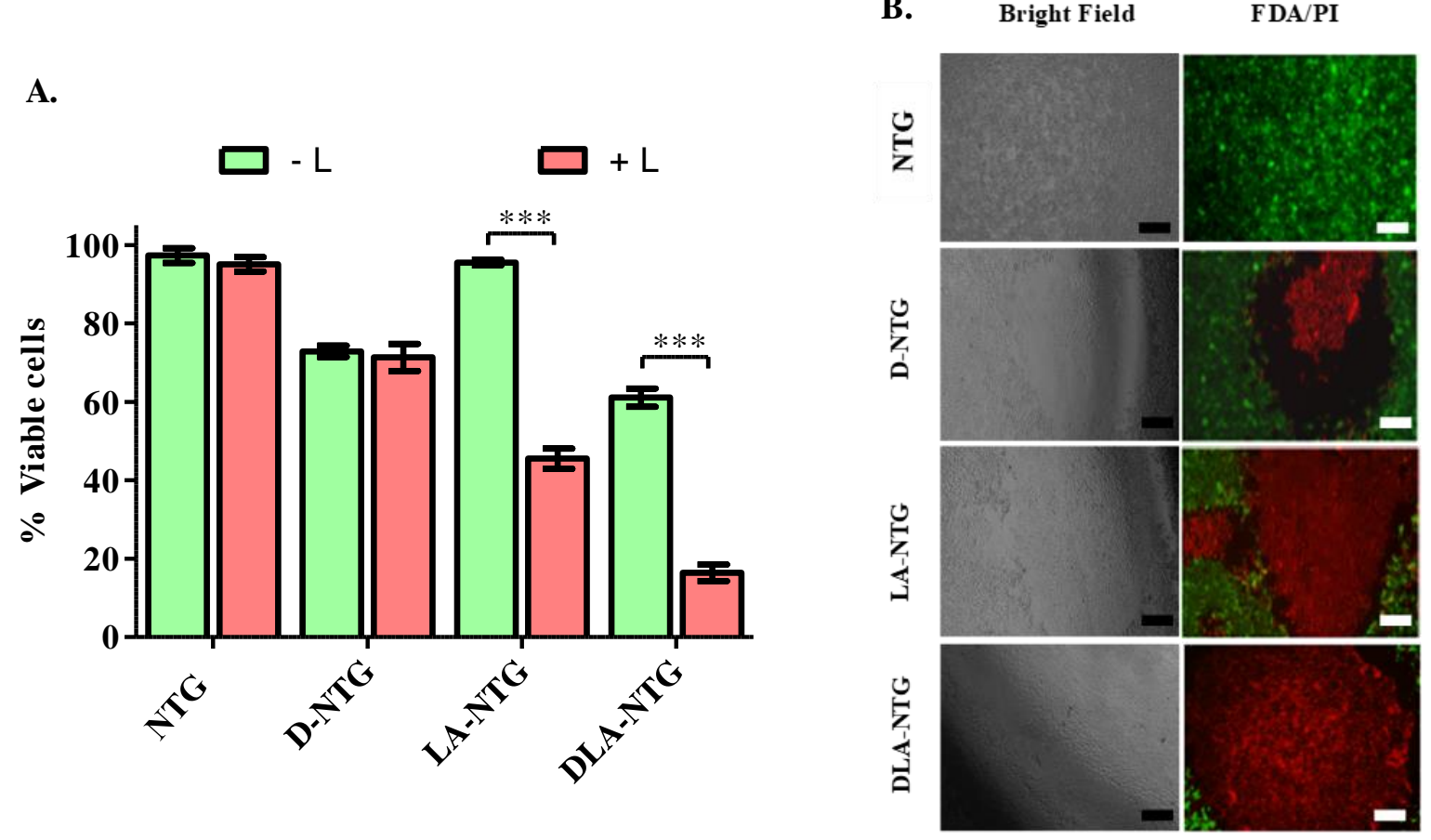

Figure S 7: A. In vitro cytotoxicity evaluation of NTG, D-NTG, LA-NTG, DLA-NTG on B16 melanoma cell line by combined chemo-photothermal therapy. B. Localized chemophotothermal therapy on B16 F10 cells assessed by FDA/PI staining. Data is representated as mean \pm SD. One-way ANOVA was performed followed by Post-Hoc Tukey's test $(* * * \mathrm{P}<0.001)$. 


\section{Supporting Information}

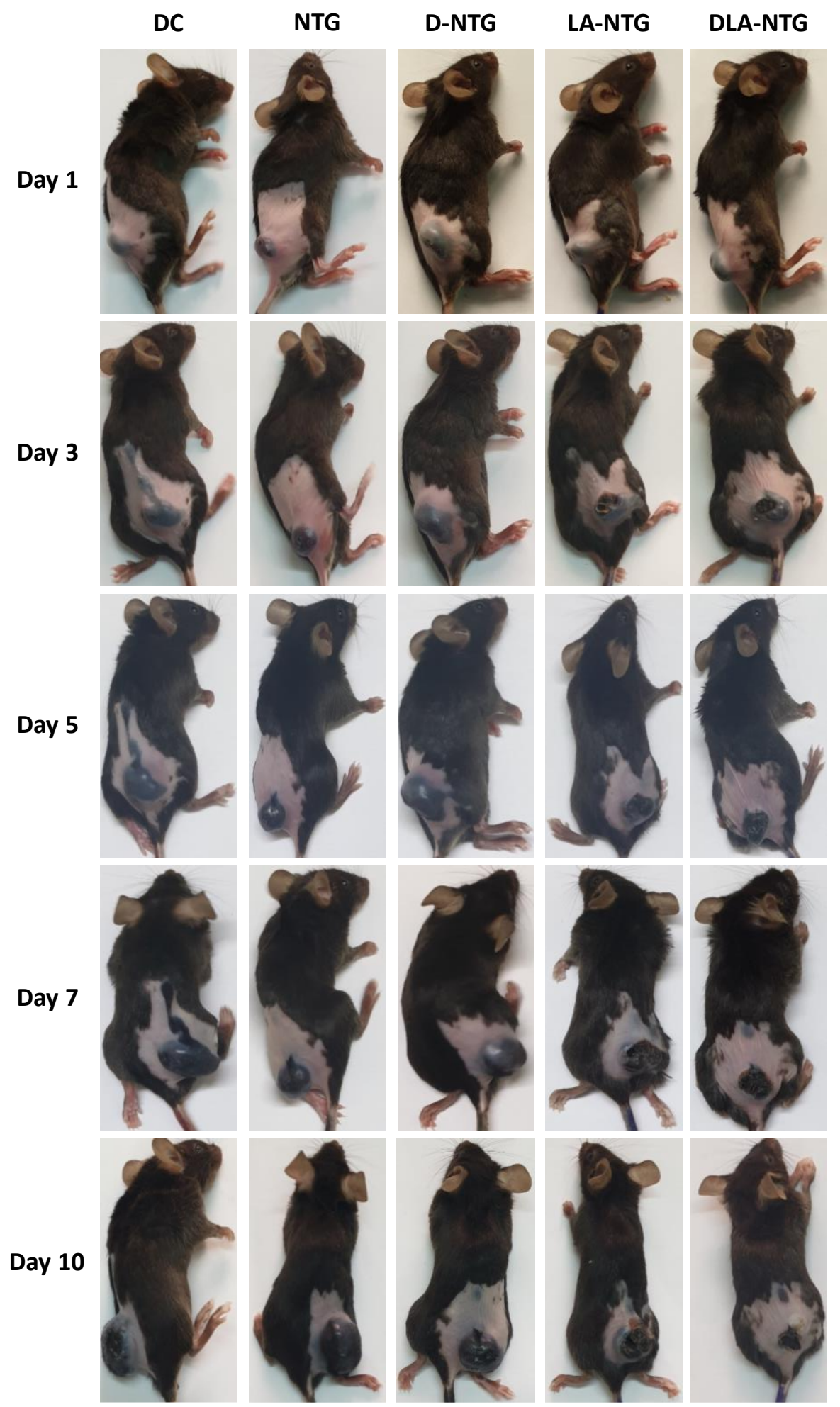

Figure S8: Tumor monitoring of B16 F10 induced melanoma during the chemo-photothermal therapy by single dose of NTG, D-NTG, LA-NTG and DLA-NTG in C5BL6 mice. 

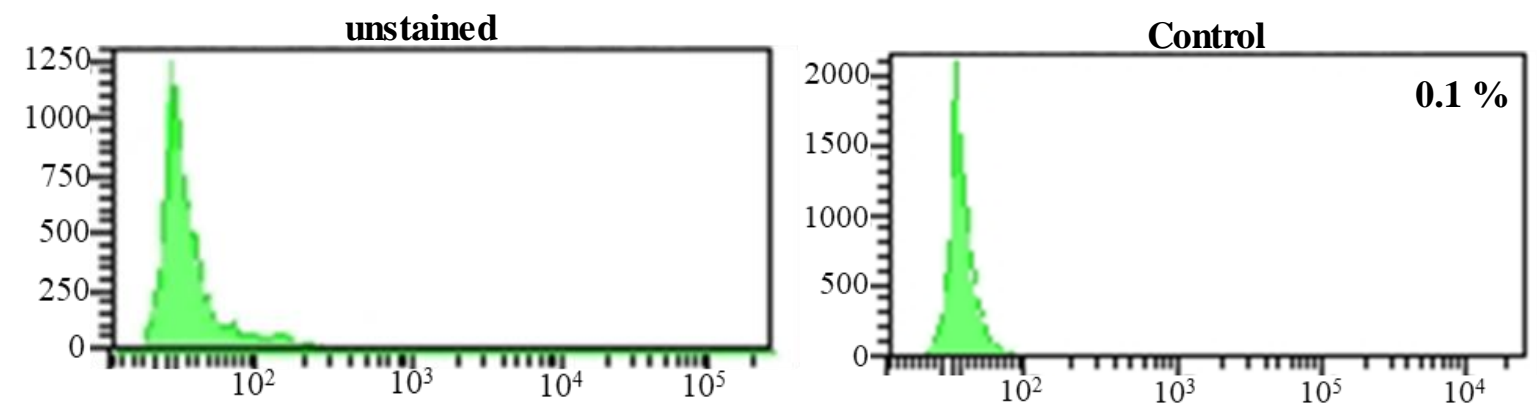

D-NTG
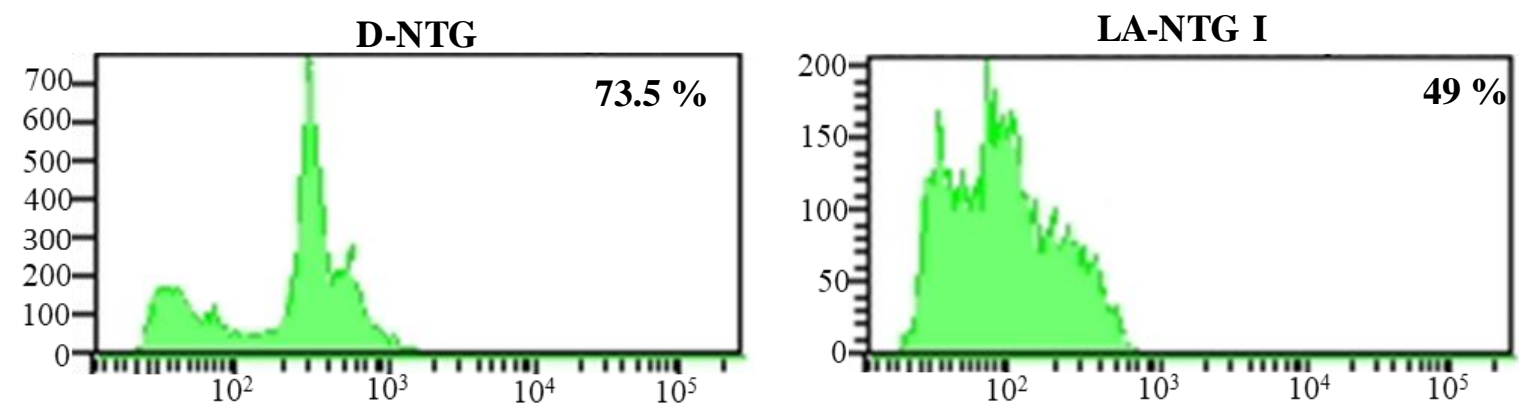

LA-NTG II

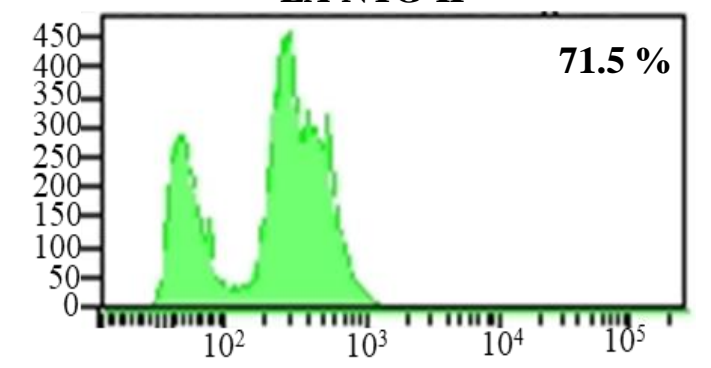

Figure S9: Intracellular uptake of D-NTG, LA-NTG I and LA-NTG II in B16F10 spheriods using flow cytometry. 
A.

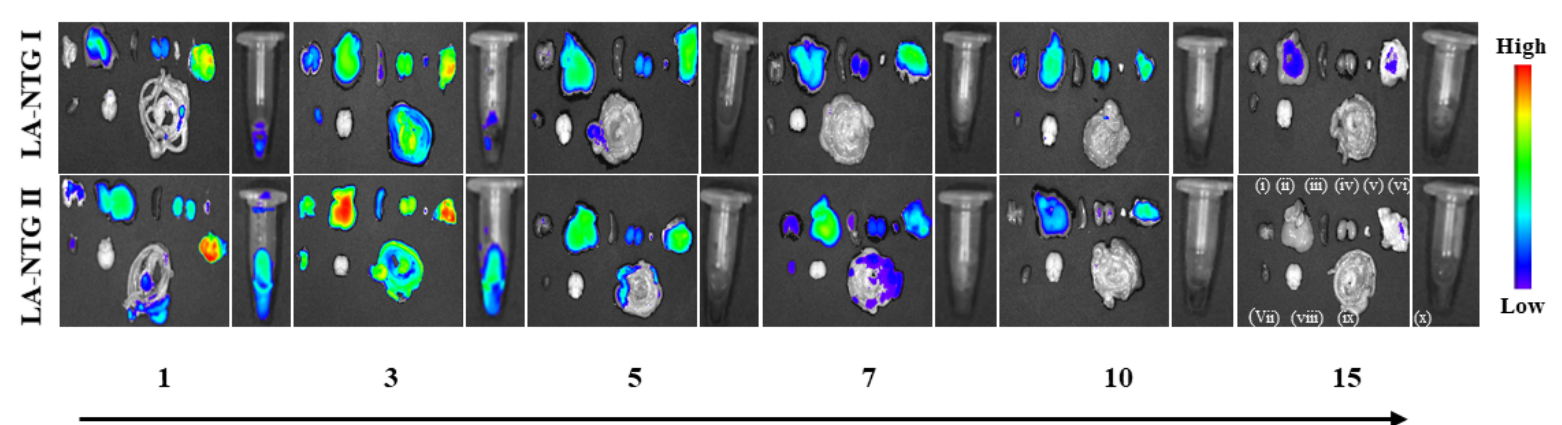

Time ( Days)

B.
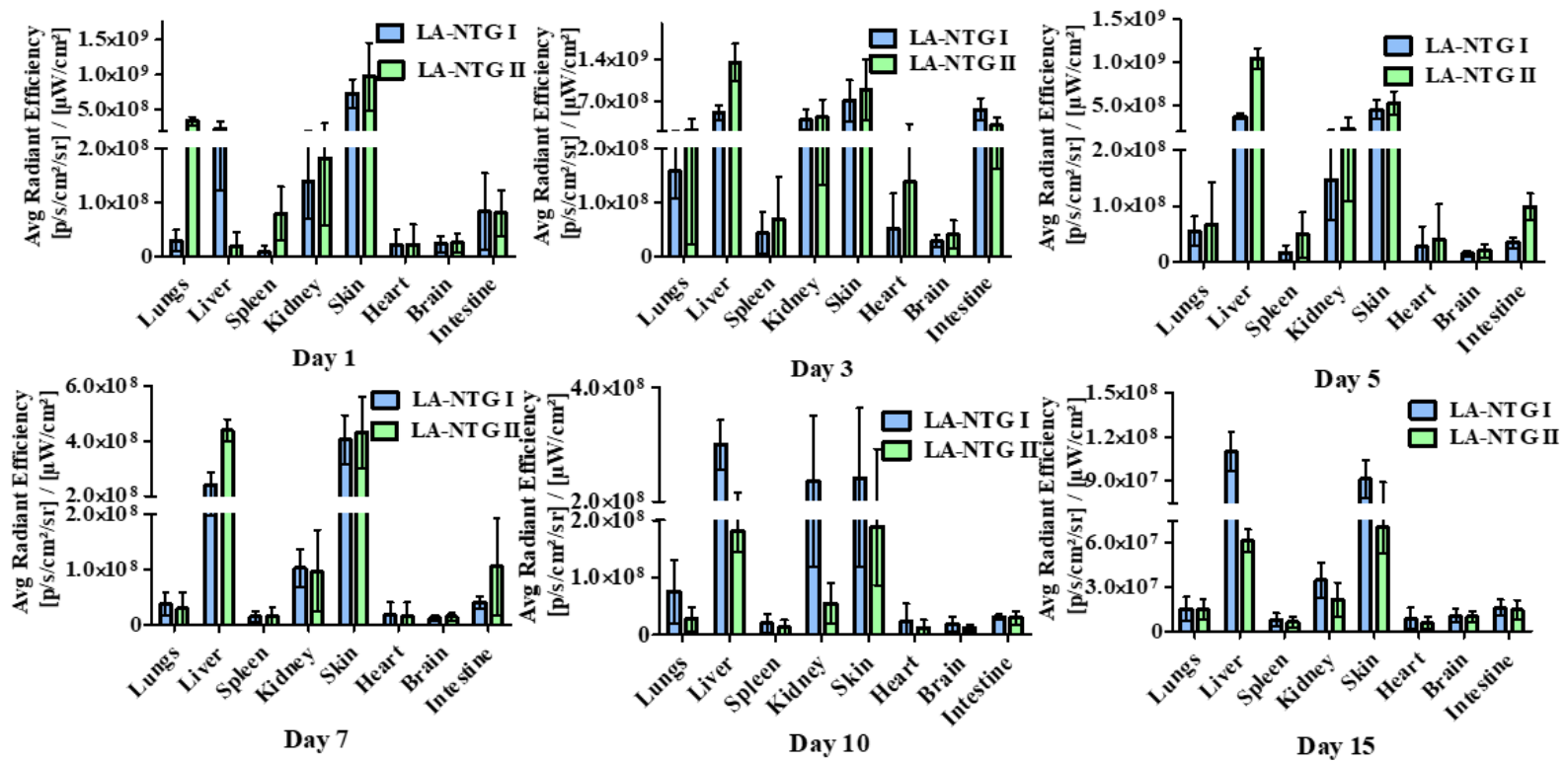

C.

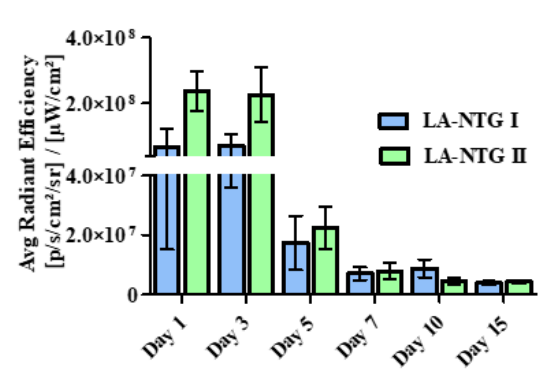

Figure S 10: A. Ex-vivo imaging of LA-NTG I and LA-NTG II tracked by IR 780 dye (i) Lungs, (ii) Liver, (iii) Spleen, (iv) Kidneys, (v) bladder, (vi) Skin, (vii) Heart (viii) Brain (ix) intestine (x) Blood. B. Biodistribution analysis of LA-NTG I and LA-NTG II at different time point (Days). C. Fluorescence signal quantification of IR 780 from LA-NTG I and LA-NTG II in blood. Data is representated as mean \pm SD. 


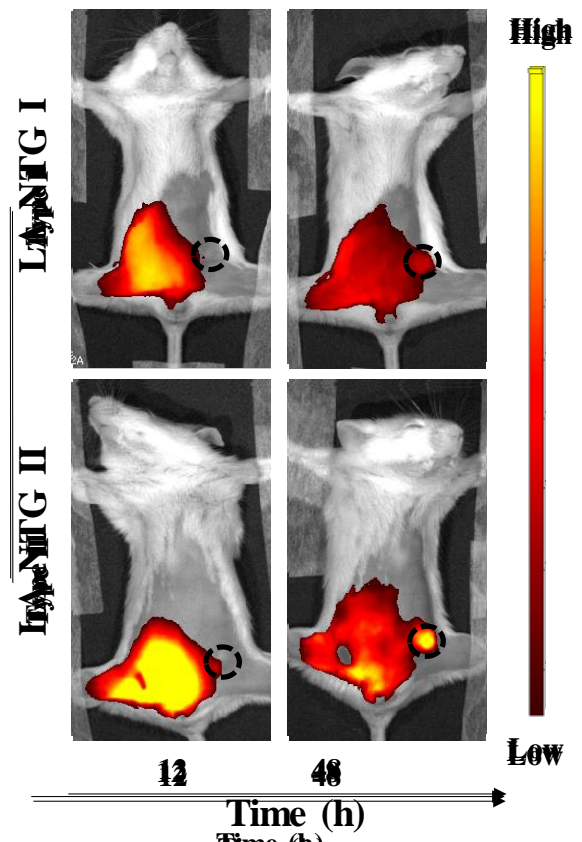

Figure S11: In vivo Single tumor targeting by LA NPs and in situ formed PA NPs released from LA-NTG as LA-NTG I and LA -NTG II respectively.

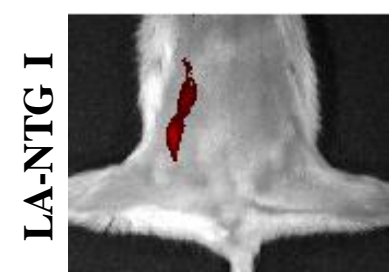

$12 \mathrm{~h}$

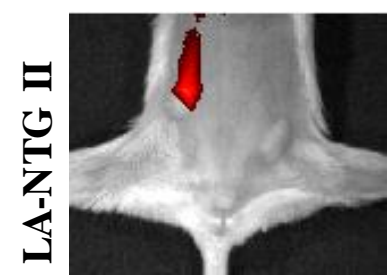

$12 \mathrm{~h}$

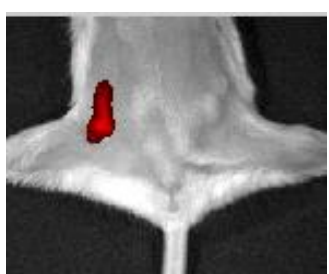

$24 \mathrm{~h}$

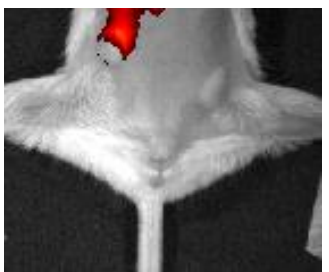

$24 \mathrm{~h}$

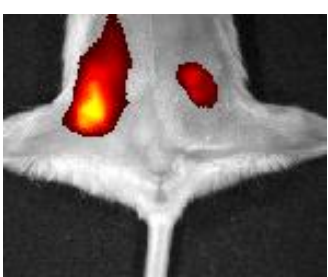

$48 \mathrm{~h}$

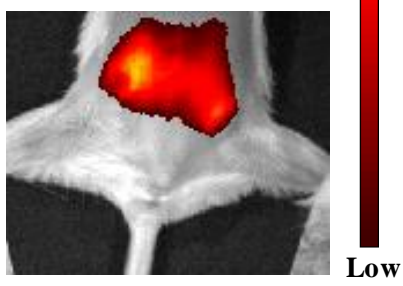

$48 \mathrm{~h}$

Figure S12: In vivo double tumor targeting by LA NPs and in situ formed PA NPs released from LA-NTG as LA-NTG I and LA -NTG II respectively. 
A.

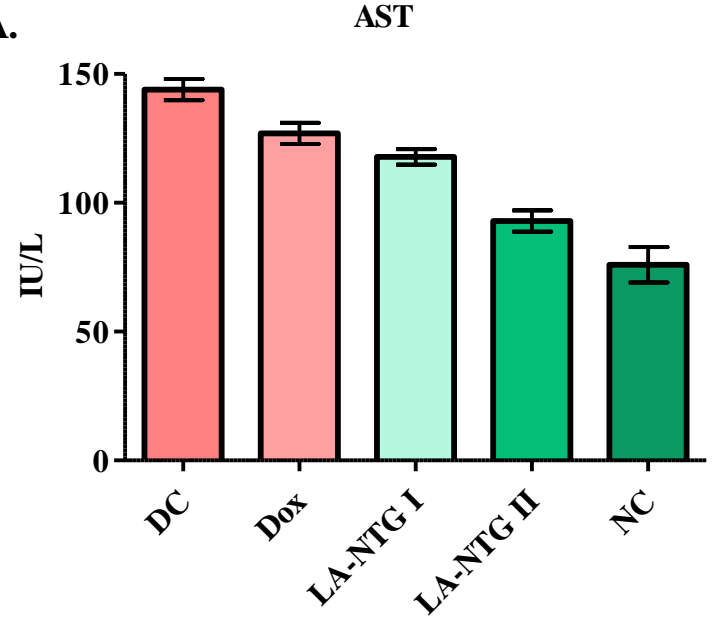

B.

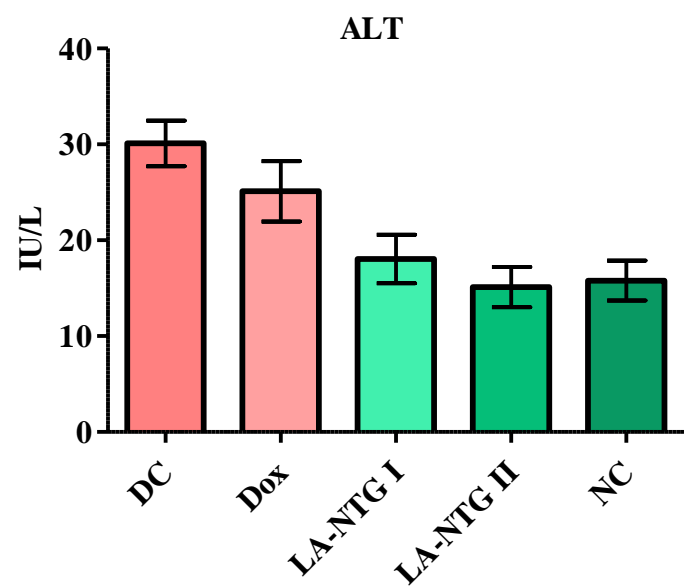

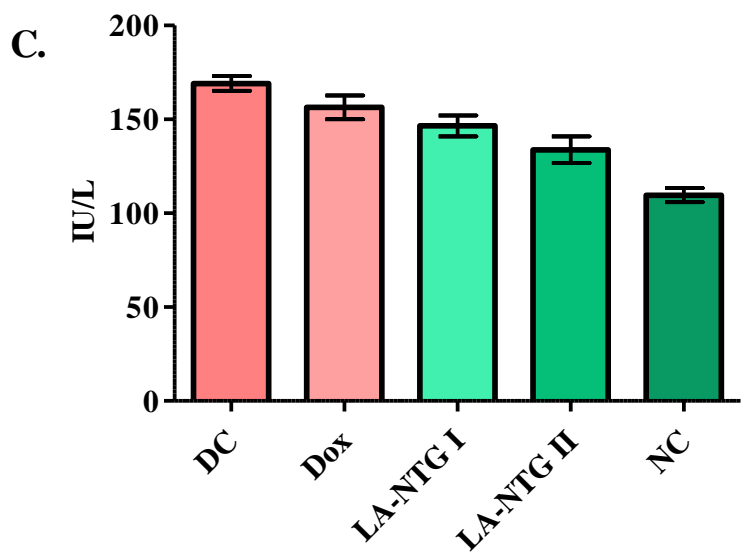

Figure S13: Serum parameter analysis ( A. AST, B. ALT and C. ALP) in disease control, Dox, LA-NTG I, LA-NTG II and normal control groups. Data is representated as mean $\pm S D(n=5)$. 